\title{
BMJ Open Relationship between environmental factors and responsive behaviours in long-term care homes: a secondary data analysis
}

\author{
Kelsey Holt (D) , ${ }^{1}$ Matthias Hoben (D) , ${ }^{2}$ Lori Weeks, ${ }^{3}$ Carole Estabrooks ${ }^{2}$
}

To cite: Holt $\mathrm{K}$, Hoben $\mathrm{M}$, Weeks L, et al. Relationship between environmental factors and responsive behaviours in long-term care homes: a secondary data analysis. BMJ Open 2021;11:e047364. doi:10.1136/ bmjopen-2020-047364

- Prepublication history for this paper is available online. To view these files, please visit the journal online (http://dx.doi. org/10.1136/bmjopen-2020047364).

Received 25 November 2020 Accepted 17 September 2021

Check for updates

(C) Author(s) (or their employer(s)) 2021. Re-use permitted under CC BY-NC. No commercial re-use. See rights and permissions. Published by BMJ.

${ }^{1}$ Faculty of Medicine, Dalhousie University, Halifax, Nova Scotia Canada

${ }^{2}$ Faculty of Nursing, University of Alberta, Edmonton, Alberta, Canada

${ }^{3}$ Faculty of Nursing, Dalhousie University, Halifax, Nova Scotia, Canada

Correspondence to

Dr Kelsey Holt;

kelsey.holt@dal.ca

\section{ABSTRACT}

Objective Responsive behaviours (eg, wandering, resisting care and verbal abuse) are a continuing issue for staff and individuals living in long-term care (LTC) homes. The LTC environment can influence responsive behaviours and is a factor in determining the quality of life for those living there. The ways in which the quality of the environment might influence responsive behaviours has not been investigated yet. We hypothesised that better quality environments would be associated with reduced rates of responsive behaviours. We used a tool that simultaneously encompasses human and structural elements of the environment, a novel approach in this field of research.

Design Cross-sectional study, using data collected from September 2014 to May 2015 as part of the Translating Research in Elder Care research programme.

Setting A representative, stratified (size, owner-operator model and health region) random sample of 76 LTC homes in British Columbia, Alberta, Manitoba.

Participants 13224 individuals (67.3\% females) living in participating LTC homes.

Outcome measures Quality of care unit work environment was assessed using the observable indicators of quality (OIQ) tool. Responsive behaviours were assessed using routinely collected Resident Assessment InstrumentMinimum Data Set V.2.0 data.

Results Adjusted regression coefficients of overall Aggressive Behaviour Scale score and interpersonal communication were $0.02(95 \% \mathrm{Cl}-0.011$ to 0.045$)$, grooming $0.06(95 \% \mathrm{Cl}-0.032$ to 0.157$)$, environmentbasics 0.067 (95\% $\mathrm{Cl} 0.024$ to 0.110 ), odour -0.066 $(95 \% \mathrm{Cl}-0.137$ to -0.004$)$, care delivery -0.007 (95\% $\mathrm{Cl}-0.033$ to 0.019$)$, environment-access -0.027 (95\% $\mathrm{Cl}-0.062$ to 0.007$)$, environment-homelike -0.034 (95\% $\mathrm{Cl}-0.065$ to -0.002$)$ and total $0 \mathrm{OQ}$ score $0.003(95 \% \mathrm{Cl}$ -0.004 to 0.010 ).

Conclusions We found small associations between the environmental quality and responsive behaviours in Western Canadian LTC homes. Higher scores on homelikeness were associated with decreased responsive behaviours. Higher scores on basic environmental quality were associated with increased responsive behaviours.
Strengths and limitations of this study

- The data used in this study are part of a wellmaintained and vast database of long-term care (LTC) homes in Western Canada, with rigorous collection standards and maintenance protocols.

- We took into account all individuals living in the LTC homes in our sample, as opposed to just those with a formal dementia diagnosis, as dementia is often under detected in LTC homes.

- The observable indicators of quality was assessed during a single window of time and communications or activities may or may not happen during this time that an observer was present.

- Observers were instructed to complete the assessment during visiting hours in common areas, which leaves out aspects of the environment where care quality could potentially vary (eg, they do not see interactions between staff and residents during morning care in a bedroom or bathroom).

\section{INTRODUCTION}

Responsive behaviours ${ }^{1}$ from people living in long-term care (LTC) homes continues to be a pervasive issue worldwide. Commonly described as behavioural and psychiatric symptoms of dementia, responsive behaviour is the preferred term by persons with dementia to represent how their actions, words and gestures are a response that expresses something important about their personal, social or physical environment. ${ }^{2}$ A systematic review identified responsive behaviour as a complex and challenging issue ${ }^{3}$ that involves multiple factors interacting in complex ways, making it difficult to address using one intervention. Thus, the causes of responsive behaviours and goal of understanding, preventing and reducing suffering for the benefit of both staff members and people living in LTC is a large area of research. ${ }^{4-13}$ Characteristics of the individuals living in LTC homes (eg, level of cognitive impairment and depression), ${ }^{14}$ as well as those of the staff members (eg, gender, 
Table 1 Covariates and justifications

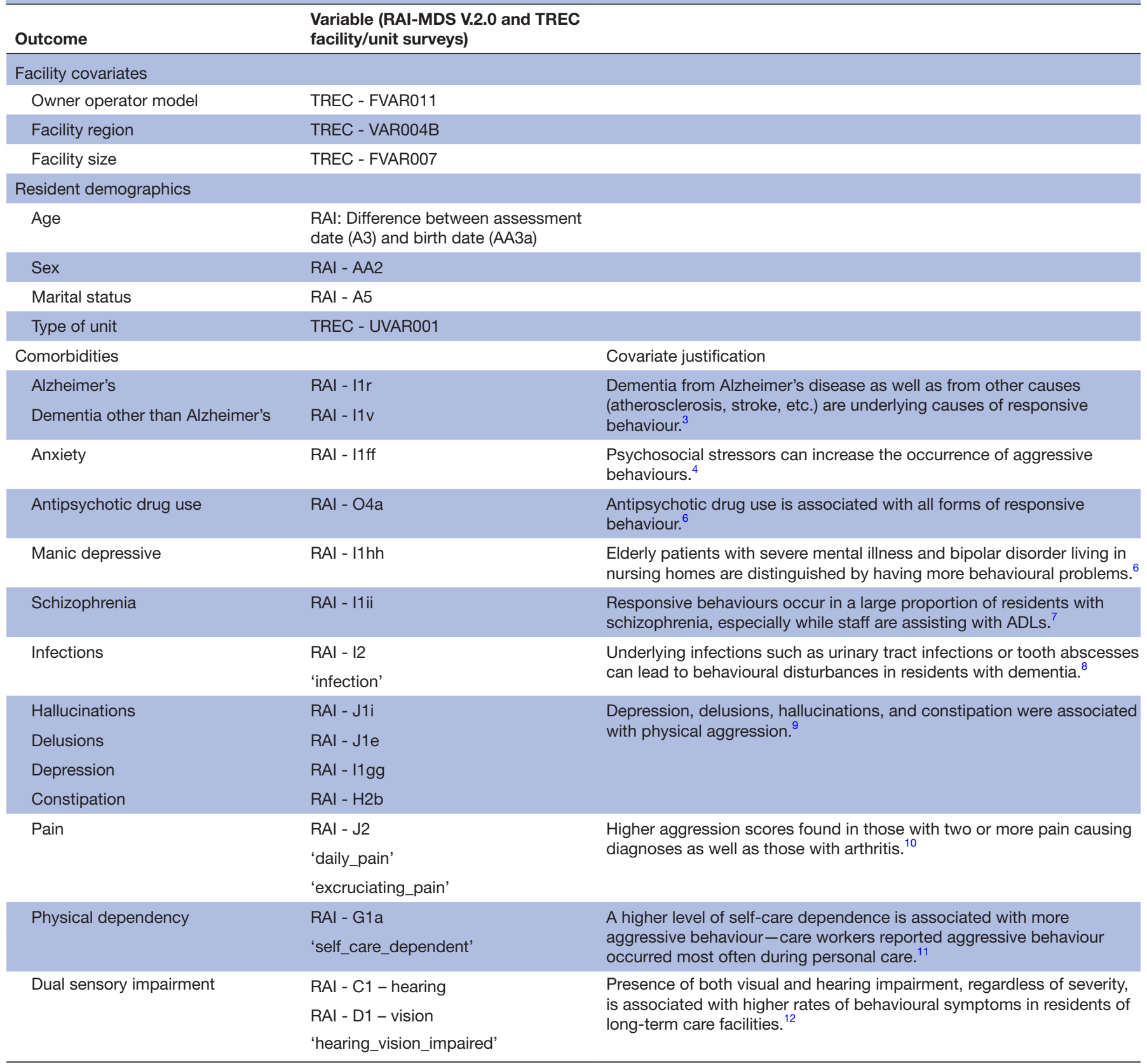

ADL, activities of daily living; RAI-MDS, Resident Assessment Instrument-Minimum Data Set; TREC, Translating Research in Elder Care.

age and training $)^{15}$ have been examined as causes, but the possible influence of environmental factors (eg, type of unit and how people communicate with each other) on responsive behaviours has received less attention. ${ }^{16}$ Focus has also been on the structural elements of the environment, such as size, homelikeness, spatial layout, noise level and temperature. ${ }^{17-19}$ However, other aspects of the environment may be important to consider. Namely, engaging environments that promote both cognitive and physical activity are associated with positive emotional responses from people with dementia. ${ }^{20}$ Additionally, the human environment (the staff who are providing care to a person) is perceived as more important than the physical environment on the quality of life for people with dementia living in LTC. ${ }^{19}$ Most recently, a 2020 study showed that higher nurse staffing and more staff psychiatric training were associated with lower prevalence of severe aggressive behaviours. ${ }^{21}$

As responsive behaviour has been shown to be influenced by multifactorial causes, it must be addressed through equally multifactorial interventions. To date, there has been less focus on the overall unit context or quality. We sought to investigate whether the quality of the LTC home is associated with responsive behaviours from individuals living in LTC homes. We hypothesised that higher quality environments would be associated with reduced rates of responsive behaviours. 
Table 2 Description of LTC home characteristics

\begin{tabular}{|c|c|c|c|c|c|c|c|c|c|c|c|c|c|}
\hline \multicolumn{14}{|l|}{ Care homes } \\
\hline & \multicolumn{2}{|c|}{ Edmonton, $\mathrm{AB}$} & \multirow{2}{*}{\multicolumn{2}{|c|}{$\begin{array}{l}\text { Calgary, AB } \\
(n=13)\end{array}$}} & \multirow{2}{*}{\multicolumn{2}{|c|}{$\begin{array}{l}\text { Fraser Health, BC } \\
(n=21)\end{array}$}} & \multirow{2}{*}{\multicolumn{2}{|c|}{$\begin{array}{l}\text { Interior Health, BC } \\
(n=13)\end{array}$}} & \multirow{2}{*}{\multicolumn{2}{|c|}{$\begin{array}{l}\text { Winnipeg, MN } \\
(n=15)\end{array}$}} & \multirow[t]{2}{*}{ P value* } & \multirow{2}{*}{\multicolumn{2}{|c|}{$\begin{array}{l}\text { Total } \\
(n=76)\end{array}$}} \\
\hline & \multicolumn{2}{|c|}{$(n=14)$} & & & & & & & & & & & \\
\hline Small ( $<80$ beds) & 3 & 21.4 & 2 & 15.4 & 6 & 28.6 & 4 & 30.8 & 2 & 13.3 & 0.071 & 17 & 22.4 \\
\hline Medium (80-120 beds) & 3 & 21.4 & 1 & 7.7 & 9 & 42.9 & 7 & 53.8 & 6 & 40 & & 26 & 34.2 \\
\hline Public not-for-profit & 2 & 14.3 & 2 & 15.4 & 4 & 19 & 5 & 38.5 & 1 & 6.7 & 0.289 & 14 & 18.4 \\
\hline Private for profit & 6 & 42.9 & 7 & 53.8 & 11 & 52.4 & 6 & 46.2 & 5 & 33.3 & & 35 & 46.1 \\
\hline Voluntary not-for-profit & 6 & 42.9 & 4 & 30.8 & 6 & 28.6 & 2 & 15.4 & 9 & 60 & & 27 & 35.5 \\
\hline \multicolumn{14}{|l|}{ Care units } \\
\hline General long-term care & 34 & 65.4 & 37 & 62.7 & 53 & 74.6 & 21 & 51.2 & 49 & 90.7 & $<0.0001$ & 194 & 68.6 \\
\hline Secure dementia & 9 & 17.3 & 17 & 28.8 & 12 & 16.9 & 11 & 26.8 & 5 & 9.3 & & 54 & 19.1 \\
\hline Non-secure dementia & 4 & 7.7 & 1 & 1.7 & 2 & 2.8 & 0 & 4.9 & 0 & 0 & & 7 & 2.5 \\
\hline $\begin{array}{l}\text { Secure mental health/ } \\
\text { psychiatric }\end{array}$ & 1 & 1.9 & 1 & 1.7 & 1 & 1.4 & 0 & 0 & 0 & 0 & & 3 & 1.1 \\
\hline Other & 4 & 7.7 & 3 & 5.1 & 3 & 4.2 & 15 & 36.6 & 0 & 0 & & 25 & 8.8 \\
\hline
\end{tabular}

${ }^{*} \mathrm{P}$ values for count data (N) from LTC homes and units reported as two-sided (asymptotic significance) calculated from Pearson $\chi^{2}$ test using $95 \%$ CI Monte Carlo simulations. $P$ values from mean values (care hours) calculated using one-way ANOVA.

ANOVA, analysis of variance; LTC, long-term care.

\section{METHODS}

\section{Study design and setting}

This study was a secondary data analysis of a subset of data from the Translating Research in Elder Care (TREC) research programme. ${ }^{22}$ TREC is a longitudinal (2007ongoing) programme of applied health services research and its aim is to improve quality of care, quality of life and quality of working life by developing and testing practical solutions to improve these outcomes. TREC's cohort of 94 LTC homes is a random sample, stratified by (a) health region (Calgary and Edmonton Zones in Alberta; Fraser and Interior Health Regions in British Columbia and Winnipeg Region Health Authority in Manitoba), (b) facility size (small, $<80$ beds; medium, 80-120 beds; large, $>120$ beds) and (c) owneroperator model (private for-profit, public not-for-profit and voluntary not-for-profit). Facility-level and unit-level data are collected using validated surveys from facility administrators, unit level data from care managers and individual level data from both regulated and unregulated staff engaged in the provision of care..$^{23}$ Informed consent is obtained from participants for this data collection on entry to the LTC home. Resident-level data are obtained quarterly from the Resident Assessment Instrument-Minimum Data Set V.2.0 (RAIMDS V.2.0).$^{22}$ Resident demographic and outcome data are deidentified at the individual resident level.

\section{Sample}

This study used a subset of data collected from September 2014 to May 2015. Our sample included 13224 residents living on 283 units in 76 LTC homes. This subset was selected as it contained the Observable Indicators of Nursing Home Care Quality (OIQ) data, a measure of the quality of the environment. ${ }^{24}$ All residents were included, as opposed to just those with a diagnosis of dementia, because responsive behaviours can be prevalent in nursing home residents with and without dementia. ${ }^{25}$ Furthermore, recent data from the Canadian Institute for Health Information suggest that almost $90 \%$ of all LTC home residents have some form of cognitive impairment, while about $70 \%$ have a diagnosis of dementia (Continuing Care Reporting System, 2015-2016, Canadian Institute for Health Information). It is possible more may be affected than are diagnosed since dementia is often under detected in LTC homes. ${ }^{26}$

\section{Outcomes and measures}

Responsive behaviour

Our measure of responsive behaviour was the RAI-MDS V.2.0 Aggressive Behaviour Scale (ABS) and one item measuring wandering. The ABS is a summary scale of the following four RAI-MDS items: verbally abusive (eg, screaming at others), physically abusive (eg, hitting others), socially inappropriate or disruptive (eg, throwing food) and resisting care (eg, pushing caregiver during activities of daily living assistance). The frequency of ABS items is coded over 7 days as not exhibited $(0)$; behaviour occurred 1-3 days in past 7 days (1); behaviour occurred 4-6 days in past 7 days, but less than daily (2) or behaviour 


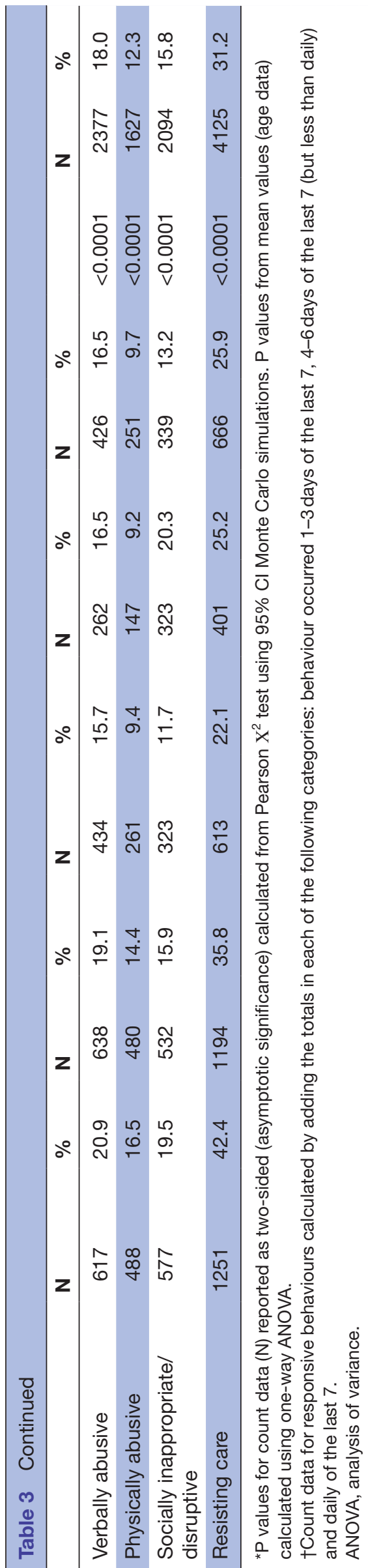

occurred daily (3). Scores for the ABS thus range from 0 to 12 , with a higher score indicating a greater number of behaviours occurring with greater frequency. The ABS has been shown to be a reliable, valid and clinically relevant measure of aggressive behaviour. ${ }^{27}$ In addition to the behaviours in the ABS, we also included wandering as a responsive behaviour in our analyses. It is coded in the same way as the ABS items described previously. Our justification for including this measure in addition to the four behaviours in the ABS is that wandering is also a responsive behaviour that has been included as such in a number of other studies looking at behaviours from people living in LTC homes. ${ }^{9} 132829$ The average inter-rater reliability estimates for all behaviour items on the RAI-MDS V.2.0 is excellent, with a $\kappa$ of $0.72 .{ }^{27}$

\section{Environmental quality}

Our measure of environmental quality was the OIQ instrument score, developed by Rantz and colleagues in $1999^{24}$ and subsequently validated. ${ }^{30}{ }^{31}$ The OIQ is a 30-item questionnaire designed for evaluating the quality of care in LTC homes via direct observation in $\leq 30 \mathrm{~min}$. Observation of behaviours is a common tool for research in this field. ${ }^{32}$ The OIQ has been used in a study to represent overall nursing home quality and has been adapted for use in Brazil and Korea since its creation. ${ }^{33-35}$ More information about the OIQ from its authors can be found online (https://nursinghomehelporg/oiq-guide/).

Study coordinators (one in each region) were trained to complete the OIQ and these study coordinators trained the data collectors (1-2 for each region)based on instructions developed by the OIQ developers. We conducted calibration exercises and inter-rater reliability assessments. To complete the OIQ, evaluators observed the unit during regular visiting hours for $5-10 \mathrm{~min}$ after reading the questionnaire and instructions. They were instructed to observe the living areas without carrying a clipboard or papers and fill out the questionnaire after observation was complete. Items on the OIQ are scored on a Likert scale from 1 to 5 . The scores from items are then grouped into the following seven first-order factors:

1. Interpersonal communication, range of 6-30 points (eg, Did residents and staff acknowledge each other and seem comfortable with each other, using smile, eye contact, touch, etc?).

2. Care delivery, 5-25 points (eg, Did staff communicate with confused residents in positive ways, eg, talk, touch, sit with, etc?).

3. Grooming, 2-10 points (eg, Were residents dressed and clean?).

4. Odour, 2-10 points (eg, Were odours of urine or faeces noticeable in the facility?).

5. Environment-basics, 5-25 points (eg, Were hallways and common areas uncluttered?).

6. Environment-access, 4-20 points (eg, Did confused residents have access to outdoor space?). 


\begin{tabular}{|c|c|c|c|c|c|c|c|c|}
\hline & $\begin{array}{l}\text { Interpersonal } \\
\text { communications } \\
\text { (6-30 points) }\end{array}$ & $\begin{array}{l}\text { Grooming } \\
(2-10 \\
\text { points) }\end{array}$ & $\begin{array}{l}\text { Environment- } \\
\text { basics (5-25 } \\
\text { points) }\end{array}$ & $\begin{array}{l}\text { Odour } \\
\text { (2-10 } \\
\text { points) }\end{array}$ & $\begin{array}{l}\text { Care delivery } \\
\text { (5-25 points) }\end{array}$ & $\begin{array}{l}\text { Environment- } \\
\text { access (4-20 } \\
\text { points) }\end{array}$ & $\begin{array}{l}\text { Environment- } \\
\text { homelike (5-25 } \\
\text { points) }\end{array}$ & $\begin{array}{l}\text { Total score } \\
(29-145 \\
\text { points) }\end{array}$ \\
\hline Median & 27 & 8 & 20 & 9 & 19 & 12 & 19 & 119 \\
\hline Mode & 30 & 8 & 20 & 10 & 20 & 12 & 20 & 110,123 \\
\hline Range & 20 & 4 & 13 & 5 & 20 & 14 & 16 & 67 \\
\hline Maximum & 30 & 10 & 25 & 10 & 25 & 19 & 25 & 141 \\
\hline \multicolumn{9}{|l|}{ Percentiles } \\
\hline 25 & 24 & 8 & 19 & 8 & 16 & 11 & 16 & 110 \\
\hline 50 & 27 & 8 & 20 & 9 & 19 & 12 & 19 & 119 \\
\hline 75 & 30 & 10 & 22 & 10 & 21 & 15 & 21 & 125 \\
\hline
\end{tabular}

$\mathrm{OIQ}$, observable indicators of quality.

7. Environment-homelike, 5-25 points (eg, Were residents' rooms personalised with furniture, pictures and other things from their past?).

We used these first-order factors and the total OIQ score (range of 29-145 points) from each facility in our analyses. Observations were collected over a 1-month period during the same time as the sample data from 2014 to 2015.

\section{Covariates}

See table 1 for all covariates and why we included them.

\section{Statistical analyses}

Descriptive analyses

All analyses were completed using SPSS V.26. For the descriptive statistics of individuals and LTC homes, differences across regions for count data (eg, number of females) were reported as two-sided asymptotic significance calculated from Pearson $\chi^{2}$ test using $95 \%$ CI Monte Carlo simulations. One-way analysis of variance (ANOVA) was used to assess differences in mean values across regions (eg, age data). Descriptive statistics for the OIQ scores across all units in our sample were generated using non-parametric techniques including minimum, maximum, mode and independent samples KruskalWallis testing to determine differences in scores across regions.

\section{Regression models}

Mixed linear regression modelling was used to assess the association between OIQ score and responsive behaviours in LTC homes. We used a backward, stepwise approach with known covariates for responsive behaviours such as depression status, pain and needing assistance with self-care based on whether they were statistically insignificant to the model fit. For a full list of the covariates that we used, see table 1 . We accounted for dependencies of data collected from residents nested within units and units nested within facilities by including random unit-level and facility-level intercepts. We also calculated intracluster correlation coefficients for unit-level and facility-level variables by dividing either the unit-level or the facility-level variance component by the total variance (sum of individual resident-level, unit-level and facilitylevel variance components). Five units $(1.8 \%)$ and the 215 individuals living in them (1.6\%) were missing data for their environment-homelike scores. As missing data were minimal, and according to Little's MCAR(Missing Completely At Random) test were indeed missing completely at random, we excluded cases with missing data from the analysis. Our mixed models do not require a strong normal assumption and inference on fixed effects is usually robust to non-normality of random effects. ${ }^{36}$

\section{RESULTS}

\section{Description of facility and sample characteristics}

Characteristics for the 76 LTC homes are found in table 2. Sample characteristics for the 13244 participants are found in table 3 .

\section{OIQ scores}

In general, units scored highly on the OIQ items (table 4), suggesting the overall quality of the environment was being rated highly by evaluators. Across all units in our sample, the modal scores were as follows: interpersonal communication $30 / 30$, grooming $8 / 10$, odour $10 / 10$, care delivery $20 / 25$, environment-basics $20 / 25$, environment-access $12 / 20$ and environment-homelike 20/25. Total OIQ score had two modal scores that were equally common-110 and 123 of 145 .

\section{Associations between responsive behaviours and OIQ scores}

Small but statistically significant associations were found between responsive behaviours and two of the OIQ scores (tables 5 and 6). Higher scores for environmenthomelike $(\mathrm{B}=-0.034, \mathrm{p}=0.036)$ were associated with decreased responsive behaviours. Higher scores for environment-basics $(\mathrm{B}=0.067, \mathrm{p}=0.024)$ were associated with increased responsive behaviours. Interpersonal communication $(\mathrm{B}=0.017, \mathrm{p}=0.221)$, grooming $(\mathrm{B}=0.063$, 
Table 5 Associations of unit-level environmental factors (OIQ scores) with resident responsive behaviours (ABS score), adjusted for facility, care unit and resident characteristics, based on hierarchical mixed models

\begin{tabular}{|c|c|c|c|}
\hline Variable & Estimate $(95 \% \mathrm{Cl})$ & Std. error & $P$ value \\
\hline Intercept & $0.09(-0.76$ to 0.95$)$ & 0.43 & 0.83 \\
\hline OIQ: Interpersonal communications & $0.02(-0.01$ to 0.05$)$ & 0.01 & 0.22 \\
\hline OIQ: Grooming & $0.06(-0.03$ to 0.16$)$ & 0.05 & 0.19 \\
\hline OIQ: Odour & $-0.07(-0.14$ to 0.004$)$ & 0.04 & 0.06 \\
\hline OIQ: Care delivery & $-0.01(-0.03$ to 0.02$)$ & 0.01 & 0.6 \\
\hline OIQ: Environment basics & 0.07 (0.02 to 0.11$)$ & 0.02 & 0.003 \\
\hline OIQ: Environment access & $-0.03(-0.06$ to 0.01$)$ & 0.02 & 0.12 \\
\hline OIQ: Environment homelike & $-0.03(-0.07$ to -0.002$)$ & 0.02 & 0.04 \\
\hline \multicolumn{4}{|l|}{ Sex $(r e f=m a l e)$} \\
\hline Female & $-0.17(-0.25$ to -0.08$)$ & 0.04 & 0 \\
\hline \multicolumn{4}{|l|}{ Marital status (ref=never married) } \\
\hline Married & $-0.05(-0.20$ to 0.11$)$ & 0.08 & 0.55 \\
\hline Widowed & $-0.01(-0.16$ to 0.13$)$ & 0.07 & 0.85 \\
\hline Separated & $-0.10(-0.32$ to 0.15$)$ & 0.12 & 0.48 \\
\hline Divorced & $0.15(-0.03$ to 0.33$)$ & 0.09 & 0.1 \\
\hline Unknown & $0.33(0.06$ to 0.60$)$ & 0.14 & 0.02 \\
\hline \multicolumn{4}{|l|}{ Bowel elimination pattern (ref=no constipation) } \\
\hline No bowel elimination & $-0.001(-0.10$ to 0.10$)$ & 0.05 & 0.98 \\
\hline Constipation & $0.22(0.00$ to 0.44$)$ & 0.11 & 0.05 \\
\hline Self care dependent (ref=not dependent) & 0.23 (0.13 to 0.32$)$ & 0.05 & 0 \\
\hline Daily pain (ref=no daily pain) & 0.31 (0.19 to 0.43$)$ & 0.06 & 0 \\
\hline Hearing and vision impairment (ref=no impairment) & $0.33(0.06$ to 0.60$)$ & 0.14 & 0.02 \\
\hline Hallucinations (ref=none) & $0.37(0.15$ to 0.59$)$ & 0.11 & 0.001 \\
\hline Delusions (ref=none) & $1.02(0.80$ to 1.24$)$ & 0.11 & 0 \\
\hline Schizophrenia $(r e f=n o)$ & $-0.65(-0.89$ to -0.40$)$ & 0.12 & 0 \\
\hline Manic depressive (ref=no) & $-0.28(-0.56$ to -0.003$)$ & 0.14 & 0.05 \\
\hline Dementia other than Alzheimer's disease (ref=none) & 0.27 (0.20 to 0.35$)$ & 0.04 & 0 \\
\hline Depression (ref=none) & $0.10(0.02$ to 0.18$)$ & 0.04 & 0.02 \\
\hline Receiving antipsychotic medication & $0.12(0.11$ to 0.14$)$ & 0.01 & 0 \\
\hline \multicolumn{4}{|l|}{ Type of unit (ref=general LTC) } \\
\hline Secure dementia & 1.08 (0.88 to 1.28$)$ & 0.1 & 0 \\
\hline Secure psychiatric & $0.21(-0.48$ to 0.91$)$ & 0.35 & 0.55 \\
\hline Other & 0.21 ( -0.08 to 0.51$)$ & 0.15 & 0.16 \\
\hline Non-secure dementia & 0.81 (0.31 to 1.31$)$ & 0.25 & 0.002 \\
\hline \multicolumn{4}{|l|}{ Ownership (ref=private for-profit) } \\
\hline Public not for profit & $0.43(0.18$ to 0.67$)$ & 0.12 & 0.001 \\
\hline Voluntary not-for-profit & $-0.16(-0.33$ to 0.02$)$ & 0.09 & 0.081 \\
\hline \multicolumn{4}{|l|}{ TREC 2 region (ref=Winnipeg) } \\
\hline Edmonton & $0.29(0.03$ to 0.55$)$ & 0.13 & 0.03 \\
\hline Calgary & $-0.22(-0.49$ to 0.04$)$ & 0.13 & 0.1 \\
\hline Frasier health & $-0.56(-0.82$ to -0.31$)$ & 0.13 & 0 \\
\hline Interior health & $-0.79(-1.10$ to -0.48$)$ & 0.15 & 0 \\
\hline
\end{tabular}


Table 5 Continued

Estimates of covariance parameters*

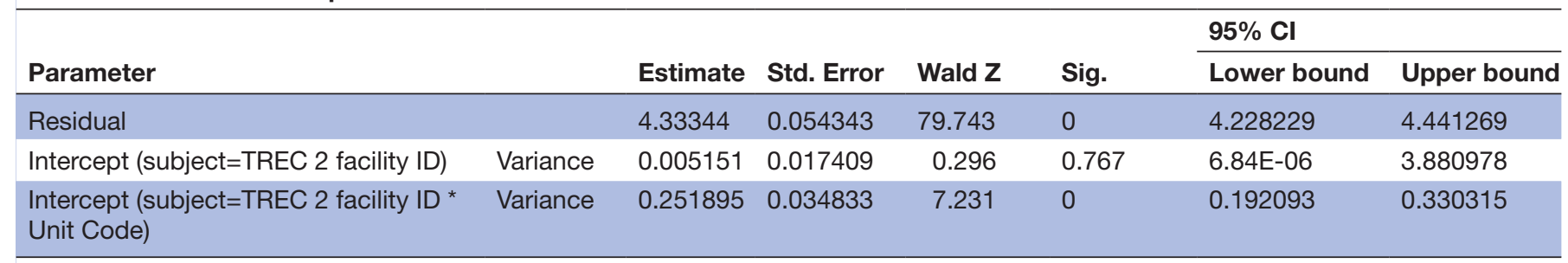

*Dependent Variable: Overall_ABS_Score.

ABS, Aggressive Behaviour Scale; OIQ, observable indicators of quality; TREC, Translating Research in Elder Care.

$\mathrm{p}=0.191$, environment-access $(\mathrm{B}=-0.027, \mathrm{p}=0.120)$, care delivery $(\mathrm{B}=-0.007, \mathrm{p}=0.566)$, odour $(\mathrm{B}=-0.066, \mathrm{p}=0.064)$ and total OIQ score $(\mathrm{B}=0.003, \mathrm{p}=0.414)$ were not associated with responsive behaviours in a statistically significant way.

Intracluster correlation coefficients (ICCs) were calculated based on each of the statistical models by dividing the unit-level variance component by the total variance (unit plus facility). For the individual OIQ scores, unitlevel ICC $=0.057$ (95\% CI 0.045 to 0.07$)$. For the facilitylevel, ICC $=0.005(95 \%$ CI 0.001 to 0.034$)$. For the total OIQ scores, unit-level ICC $=0.055$ (95\% CI 0.044 to 0.068 ) and facility-level ICC=0.007 (95\% CI 0.002 to 0.028$)$.

\section{DISCUSSION}

Only two of the seven OIQ scores were associated at statistically significant levels with responsive behaviours. A more homelike atmosphere was associated with decreased responsive behaviours. This is consistent with existing research. For example, Chaudhury et $a l^{17}$ reported that having a more homelike environment reduces responsive behaviours such as wandering. Higher basic environment quality scores (hallways free from clutter, well lit, etc) were associated with more responsive behaviours. This could be explained by the fact we included wandering as a responsive behaviour in our analysis, and there is a higher likelihood for wandering to occur in areas that are brightly lit as well as hallways. ${ }^{28} 37$

We found no association between having access to safe spaces to wander and the outdoors and responsive behaviours, despite previous literature that showed this reduces wandering behaviours in LTC homes. ${ }^{1737}$ Higher scores on interpersonal communication were not associated with decreased responsive behaviours, although optimised communication is a well described strategy for reducing responsive behaviours, specifically communicating empathetically and adopting a person-centred approach. ${ }^{38}$ These elements are part of the interpersonal communication section of the OIQ (eg, Did staff appear caring/compassionate/warm/kind?). Better scores on odour were not associated with reduced responsive behaviours although odours from faeces, urine, chemicals and food have been reported to be an important antecedent condition for 'catastrophic reactions' in people with dementia. $^{39}$

We expected that in a setting where residents are better groomed, they may have staff in their personal space more often for help with hygiene, and would have higher rates of responsive behaviours. This is because entering someone's personal space, such as during times of assisting a person with toileting or personal hygiene, is known to be a trigger for increased responsive behaviours. ${ }^{5}$ Despite this we did not find a statistically significant association between grooming scores and responsive behaviours. Finally, the care delivery scores were also not significantly associated with responsive behaviours. This is unexpected because a component of the care delivery OIQ score encompasses staff communicating with confused residents/helping residents move about the facility, which as mentioned previously would put them into someone's personal space and possibly trigger responsive behaviours. ${ }^{5}$

The findings of this study suggest that including individually tailored items that make a person feel at home could reduce responsive behaviours, and that responsive behaviours are associated with cleaner, clutter-free environments, possibly because these areas are more accessible for people who wander. Ensuring a homelike environment is a feasible step that the people living in LTC homes can take, as well as their caregivers and facility management bodies. We did not find any other significant associations between environmental quality and responsive behaviours. One explanation could be that we are not capturing an important aspect of the environment in our analysis-the work environment. Lack of time was identified as barrier to getting to know patients and providing person-centred service. ${ }^{40}$ Additionally, having a team-based approach to identify underlying needs of residents is effective to reduce agitated behaviour. ${ }^{41}$ Both studies provide evidence that adequate levels of staffing and ample time for staff to provide tailored care for residents ${ }^{42}$ is a way to decrease responsive behaviours, something that our study did not consider. Examining the quality of the work environment would be an important avenue for future research in this field. 
Table 6 Associations of unit-level environmental factors (total OIQ score) with resident responsive behaviours (ABS score), adjusted for facility, care unit and resident characteristics, based on hierarchical mixed models

\begin{tabular}{|c|c|c|c|}
\hline Variable & Estimate(95\% Cl) & Std. error & $P$ value \\
\hline Intercept & $0.33(-0.51$ to 1.17$)$ & 0.43 & 0.44 \\
\hline Total OIQ score & $0.003(-0.004$ to 0.009$)$ & 0.003 & 0.41 \\
\hline Female & $-0.17(-0.25$ to -0.08$)$ & 0.04 & 0 \\
\hline \multicolumn{4}{|l|}{ Marital status (ref=never married) } \\
\hline Separated & $-0.10(-0.34$ to 0.14$)$ & 0.12 & 0.42 \\
\hline Divorced & $0.15(-0.03$ to 0.33$)$ & 0.09 & 0.11 \\
\hline Unknown & 0.34 (0.08 to 0.61$)$ & 0.14 & 0.01 \\
\hline Self care dependent (ref=not dependent) & $0.24(0.14$ to 0.34$)$ & 0.05 & 0 \\
\hline Delusions (ref=none) & $1.03(0.81$ to 1.25$)$ & 0.11 & 0 \\
\hline Schizophrenia $(r e f=n o)$ & $-0.65(-0.89$ to -0.40$)$ & 0.13 & 0 \\
\hline Manic depressive $(r e f=n o)$ & $-0.27(-0.55$ to 0.01$)$ & 0.14 & 0.06 \\
\hline Dementia other than Alzheimer's disease (ref=none) & 0.27 (0.20 to 0.35$)$ & 0.04 & 0 \\
\hline Depression (ref=none) & $0.10(0.01$ to 0.17$)$ & 0.04 & 0.03 \\
\hline \multicolumn{4}{|l|}{ Anxiety (ref=none) } \\
\hline NA & $-0.02(-0.12$ to 0.07$)$ & 0.05 & 0.64 \\
\hline Anxiety & $0.12(-0.07$ to 0.31$)$ & 0.1 & 0.22 \\
\hline Receiving antipsychotic medication & $0.12(0.11$ to 0.14$)$ & 0.01 & 0 \\
\hline Public not for profit & $0.39(0.14$ to 0.65$)$ & 0.13 & 0.003 \\
\hline Voluntary not for profit & $-0.12(-0.32$ to 0.07$)$ & 0.1 & 0.21 \\
\hline \multicolumn{4}{|l|}{ TREC 2 region (ref=Winnipeg) } \\
\hline Edmonton & 0.37 (0.08 to 0.66$)$ & 0.14 & 0.01 \\
\hline Calgary & $-0.15(0.44$ to 0.14$)$ & 0.13 & 0.29 \\
\hline Frasier health & $-0.42(-0.68$ to -0.15$)$ & 0.13 & 0.003 \\
\hline Interior health & $-0.63(-0.95$ to -0.31$)$ & 0.16 & 0 \\
\hline
\end{tabular}

\section{Estimates of covariance parameters*}

\begin{tabular}{|c|c|c|c|c|c|c|c|}
\hline \multirow[b]{2}{*}{ Parameter } & & \multirow[b]{2}{*}{ Estimate } & \multirow[b]{2}{*}{ Std. Error } & \multirow[b]{2}{*}{ Wald Z } & \multirow[b]{2}{*}{ Sig. } & \multicolumn{2}{|l|}{$95 \% \mathrm{Cl}$} \\
\hline & & & & & & Lower bound & Upper bound \\
\hline Residual & & 4.37981 & 0.054475 & 80.401 & 0 & 4.274333 & 4.487891 \\
\hline $\begin{array}{l}\text { Intercept (subject=TREC } 2 \\
\text { facility ID * Unit Code) }\end{array}$ & Variance & 0.256778 & 0.036006 & 7.131 & 0 & 0.195074 & 0.337999 \\
\hline
\end{tabular}

*Dependent variable: Overall_ABS Score.

ABS, Aggressive Behaviour Scale; OIQ, observable indicators of quality; TREC, Translating Research in Elder Care. 
The findings of this article are important for potentially changing clinical practices since it focuses on something modifiable - the care unit environment as measured by the OIQ. The TREC programme has largely measured the work environment using the Alberta Context Tool (https://trecresearch.ca/alberta_context_tool), and the OIQ is a complementary and slightly different tool to measure unit context. Given the complexity involved with studying responsive behaviours in LTC homes, where care teams and residents interact and where quality of care is created, measures of the overall unit context such as with the OIQ will prove helpful to build on our knowledge base.

\section{Limitations}

Our study was cross-sectional, so no causal conclusions from the associations can be drawn. Another limitation is that there is a ceiling effect when using the ABS as reports are limited to a score of 3 (behaviour occurred daily), so behaviours that are persistently expressed or repeated multiple times a day are not captured. It is possible given this ceiling effect that we were unable to capture those associations between more persistent responsive behaviours and the environmental quality.

The OIQ data also has limitations; first, it depends on individual observers doing data collection during a single window of time. Communications or activities may or may not happen during this time that an observer was present. Observers were instructed to complete the assessment during visiting hours in common areas, which leaves out aspects of the environment where care quality could potentially vary (eg, they do not see interactions between staff and residents during morning care in a bedroom or bathroom).

\section{Conclusion}

We found two small associations between the environmental quality and responsive behaviours in a diverse population of people living in Western Canadian LTC homes. We used a tool that simultaneously encompasses human and structural elements of the environment, a novel approach in this field of research. Specifically, higher scores on homelikeness were associated with decreased responsive behaviours. Higher scores on basic environmental quality were associated with increased responsive behaviours. Future research could examine other potential influencers of responsive behaviours in LTC, such as staff rushing tasks, and should include measures of responsive behaviours that reflect the diversity with which responsive behaviours are expressed.

Acknowledgements We acknowledge everyone involved in the TREC team for their ongoing support of this study.

Contributors $\mathrm{KH}, \mathrm{LW}$ and $\mathrm{MH}$ conceived this study. $\mathrm{KH}$ prepared, compiled and completed statistical analysis of data under guidance of MH. Joseph Akinlawon also assisted with statistical analysis. KH wrote the manuscript. CE, LW and MH reviewed and approved the final version of the manuscript.

Funding This study was supported by a Research in Medicine (RIM) stipend from Dalhousie University, specifically the Gladys 0sman Studentship. This is a
CAD\$5000.00 stipend provided to students working on their RIM project over the summer months.

Competing interests None declared.

Patient and public involvement Patients and/or the public were not involved in the design, or conduct, or reporting, or dissemination plans of this research.

Patient consent for publication Not applicable.

Ethics approval Ethics approval for the secondary analysis of this data was obtained by Dalhousie University's Health Sciences Research Ethics Board (2018-4503) and prior approval for data collection from the University of Alberta Research Ethics Board (Pro00037937), the University of British Columbia Office of Research Ethics (H14-00942) and the University of Manitoba Research Ethics Board (H2014:164 (HS17655)). For TREC survey data collection, operational approvals were obtained from the participating organisations.

Provenance and peer review Not commissioned; externally peer reviewed.

Data availability statement Data are available upon reasonable request. The data generated and analysed in this study are not publicly available due to ethical regulations around accessing the TREC database. Data could be made available to reasonable requests based on approval from TREC.

Open access This is an open access article distributed in accordance with the Creative Commons Attribution Non Commercial (CC BY-NC 4.0) license, which permits others to distribute, remix, adapt, build upon this work non-commercially, and license their derivative works on different terms, provided the original work is properly cited, appropriate credit is given, any changes made indicated, and the use is non-commercial. See: http://creativecommons.org/licenses/by-nc/4.0/.

\section{ORCID iDs}

Kelsey Holt http://orcid.org/0000-0002-1005-6783

Matthias Hoben http://orcid.org/0000-0003-3465-315X

\section{REFERENCES}

1 Dupuis SL, Wiersma E, Loiselle L. Pathologizing behavior: meanings of behaviors in dementia care. J Aging Stud 2012;26:162-73.

2 Alzheimer Society of Canada. Responsive and reactive behaviours, 2020. Available: https://alzheimer.ca/en/help-support/im-caringperson-living-dementia/understanding-symptoms/responsivereactive-behaviours

3 Holst A, Skär L. Formal caregivers' experiences of aggressive behaviour in older people living with dementia in nursing homes: A systematic review. Int J Older People Nurs 2017;12:e12158-12.

4 Majić T, Pluta JP, Mell T, et al. Correlates of agitation and depression in nursing home residents with dementia. Int Psychogeriatr 2012;24:1779-89.

5 Zeller A, Hahn S, Needham I, et al. Aggressive behavior of nursing home residents toward caregivers: a systematic literature review. Geriatr Nurs 2009;30:174-87.

6 Talerico KA, Evans LK, Strumpf NE. Mental health correlates of aggression in nursing home residents with dementia. Gerontologist 2002;42:169-77.

7 Bartels SJ, Mueser KT, Miles KM. A comparative study of elderly patients with schizophrenia and bipolar disorder in nursing homes and the community. Schizophr Res 1997;27:181-90.

8 Brodaty H, Donkin M. Family caregivers of people with dementia. Dialogues Clin Neurosci 2009;11:217-28.

9 Desai AK, Grosserg GT. Recognition and management of behavioral disturbances in dementia. Prim Care Companion J Clin Psychiatry 2001;03:93-109.

10 Leonard R, Tinetti ME, Allore HG, et al. Potentially modifiable resident characteristics that are associated with physical or verbal aggression among nursing home residents with dementia. Arch Intern Med 2006;166:1295-300.

11 Feldt KS, Warne MA, Ryden MB. Examining pain in aggressive cognitively impaired older adults. J Gerontol Nurs 1998;24:14-22.

12 Schreiner AS. Aggressive behaviors among demented nursing home residents in Japan. Int J Geriatr Psychiatry 2001;16:209-15.

13 Yamada Y, Denkinger MD, Onder G, et al. Impact of dual sensory impairment on onset of behavioral symptoms in European nursing homes: results from the services and health for elderly in long-term care study. J Am Med Dir Assoc 2015;16:329-33.

14 Wharton TC, Ford BK. What is known about dementia care recipient violence and aggression against caregivers? J Gerontol Soc Work 2014;57:460-77. 
15 Zeller A, Dassen T, Kok G, et al. Factors associated with resident aggression toward caregivers in nursing homes. J Nurs Scholarsh 2012;44:249-57.

16 Stutte K, Hahn S, Fierz K, et al. Factors associated with aggressive behavior between residents and staff in nursing homes. Geriatr Nurs 2017;38:398-405.

17 Chaudhury $\mathrm{H}$, Cooke $\mathrm{HA}$, Cowie $\mathrm{H}$, et al. The influence of the physical environment on residents with dementia in long-term care settings: a review of the empirical literature. Gerontologist 2018;58:e325-37.

18 Garre-Olmo J, López-Pousa S, Turon-Estrada A, et al. Environmental determinants of quality of life in nursing home residents with severe dementia. J Am Geriatr Soc 2012;60:1230-6.

19 Garcia LJ, Hébert M, Kozak J, et al. Perceptions of family and staff on the role of the environment in long-term care homes for people with dementia. Int Psychogeriatrics 2012;24:753-65.

20 Lee $\mathrm{KH}$, Boltz $\mathrm{M}$, Lee $\mathrm{H}$, et al. Is an engaging or soothing environment associated with the psychological well-being of people with dementia in long-term care? J Nurs Scholarsh 2017;49:135-42.

21 Orth J, Li Y, Simning A, et al. Severe behavioral health manifestations in nursing homes: associations with service availability? J Am Geriatr Soc 2020;68:2643-9.

22 Estabrooks CA, Squires JE, Cummings GG, et al. Study protocol for the translating research in elder care (TREC): building context - an organizational monitoring program in long-term care project (project one). Implement Sci 2009;4:1-13.

23 Estabrooks CA, Hutchinson AM, Squires JE, et al. Translating research in elder care: an introduction to a study protocol series. Implement Sci 2009;4:1-8.

24 Rantz MJ, Zwygart-Stauffacher M, Popejoy L, et al. Nursing home care quality: a multidimensional theoretical model integrating the views of consumers and providers. J Nurs Care Qual 1999;14:16-37.

25 Choi H, Jung Y-I, Kim H. Factors related to aggressive behaviors among older adults in nursing homes of Korea: a cross-sectional survey study. Int J Nurs Stud 2018;88:9-15.

26 Maslow K, Fortinsky RH. Nonphysician care providers can help to increase detection of cognitive impairment and encourage diagnostic evaluation for dementia in community and residential care settings. Gerontologist 2018;58:S20-31.

27 Perlman CM, Hirdes JP. The aggressive behavior scale: a new scale to measure aggression based on the minimum data set. $J$ Am Geriatr Soc 2008;56:2298-303.
28 Yao L, Algase D. Environmental ambiance as a new window on wandering. West $J$ Nurs Res 2006;28:89-104.

29 Kiely DK, Morris JN, Algase DL. Resident characteristics associated with wandering in nursing homes. Int J Geriatr Psychiatry 2000;15:1013-20.

30 Aud MA, Rantz MJ, Zwygart-Stauffacher M, et al. Developing a residential care facility version of the observable indicators of nursing home care quality instrument. J Nurs Care Qual 2004;19:48-57.

31 Rantz MJ, Zwygart-Stauffacher M, Mehr DR, et al. Field testing, refinement, and psychometric evaluation of a new measure of nursing home care quality. J Nurs Meas 2006;14:129-48.

32 Curyto KJ, Van Haitsma K, Vriesman DK. Direct observation of behavior: a review of current measures for use with older adults with dementia. Res Gerontol Nurs 2008;1:52-76.

33 Goodson J, Jang W, Rantz M. Nursing home care quality: insights from a Bayesian network approach. Gerontologist 2008;48:338-48.

34 de OWIF, Hernández PJS, Sousa K de M, et al. Equivalência semântica, conceitual $\mathrm{E}$ de itens do observable indicators of nursing home care quality instrument. Cien Saude Colet 2016;21:2243-56.

35 Lee J. Validity and reliability of the Korean version of the observable indicators of nursing home care quality evaluation instrument. $J$ Korean Acad Nurs 2008;38:474.

36 Zhang D, Davidian M. Linear mixed models with flexible distributions of random effects for longitudinal data. Biometrics 2001;57:795-802.

37 Algase DL, Beattie ERA, Antonakos C, et al. Wandering and the physical environment. Am J Alzheimers Dis Other Demen 2010;25:340-6.

38 Zeller A, Müller M, Needham I, et al. Dealing with aggressive behaviour in nursing homes: caregivers' use of recommended measures. J Clin Nurs 2014;23:2542-53.

39 Yeom H-A, Watson NM. Patterns of antecedents of catastrophic reactions in nursing home residents with dementia in the United States. Asian Nurs Res 2009;3:99-110.

40 Clifford C, Doody O. Exploring nursing staff views of responsive behaviours of people with dementia in long-stay facilities. J Psychiatr Ment Health Nurs 2018;25:26-36.

41 Gillis K, Lahaye H, Dom S, et al. A person-centred team approach targeting agitated and aggressive behaviour amongst nursing home residents with dementia using the senses framework. Int J Older People Nurs 2019;14:1-9.

42 Nybakken S, Strandås M, Bondas T. Caregivers' perceptions of aggressive behaviour in nursing home residents living with dementia: A meta-ethnography. J Adv Nurs 2018;74:2713-26. 